\title{
Measuring the Oil Weight of Crude Oil Storage Tanks Based on the Density Method
}

\author{
Chen Bo ${ }^{1, a}$, Xue Xiaoqing ${ }^{2, b}$ Liu Ting $^{3, c}$ \\ ${ }^{1}$ Physics and Electronic Engineering,Xianyang Normal University, China \\ ${ }^{1}$ Physics and Electronic Engineering,Xianyang Normal University, China \\ ${ }^{1}$ Physics and Electronic Engineering,Xianyang Normal University, China \\ Achen_bo_16@163.coml, b136465361@qq.com, cwhiteliuting@163.com
}

Keywords: crude oil measurement; differential pressure transmitter; ultrasonic sensors: crude oil moisture;

\begin{abstract}
The measurement of crude oil production of crude oil storage tanks has been a hot issue in the petrochemical industry, while crude oil storage tanks usually contain oil, water, oil and water mixture, as well as sediment and other media. Domestic measurement methods generally have poor stability, low accuracy and most of them can not realize the level measurement of multiphase media within the tank, this paper presents that the weight of crude oil can be measured based on the density method, the density value of oil and water mixture can be obtained by differential pressure transmitter and ultrasonic level sensor, thereby the crude oil production accurately in the tank could be calculated precisely.
\end{abstract}

\section{Introduction}

Since water injection method is widely used in oil exploration in the petrochemical industry, so the crude oil explored contains much moisture and impurities and need to stand in a crude oil storage tank, under the action of gravity, there are natural gas layer, oil layer, aqueous layer and impurity layer ${ }^{[1]}$. Thus, how to measure the liquid level of each of medium the dielectric layer to accurately calculate crude oil production is a research hotspot at home. Currently, the most commonly used domestic measurement methods include scale artificial methods, mechanical float method and radar method, some of these measurements can not achieve the measurement of level height of the multiphase medium within the tank, while some are costly or less efficient, so this paper studies how to measure accurately the weight of crude oil in the tank by density method ${ }^{[2]}$.

\section{Measuring Principle:}

In the crude oil storage tank, if the total volume of oil-water is $\mathrm{V}$, the volume of crude oil with water in storage tanks is $V_{w}$, the volume of pure oil in storage tanks is $V_{o}$. Suppose in the case of oil and water incompatibility, then

$$
V=V_{o}+V_{w}
$$

According to the law of conservation of mass, then

$$
\rho_{m} V=\rho_{o} V_{o}+\rho_{w} V_{w}
$$

Where:

$$
\begin{aligned}
& \rho_{m} \text { stands for the mixed density of the aqueous crude; } \\
& \rho_{o} \text { stands for the density of pure oil; } \\
& \rho_{w} \text { stands for water density. }
\end{aligned}
$$

Deform formula (1) into $V_{o}=V-V_{w}$ and take it into formula (2), then comes 


$$
\alpha=\frac{V_{w}}{V}=\frac{\rho_{m}-\rho_{o}}{\rho_{w}-\rho_{o}}
$$

Where:

$\alpha$ stands for Volumetric water content in crude oil storage tanks.

From equation (3), the weight of pure oil in crude oil storage tanks can be inferred

$$
M_{o}=V(1-\alpha) \rho_{o}
$$

Where:

$M_{o}$ stands for the weight of crude oil in storage tanks.

Crude oil storage tanks in this article are all standard, namely cylindrical tanks. If the cross-sectional area for crude oil storage tank is $\mathrm{S}$, tank liquid level is $\mathrm{H}$, then

$M_{o}=H S(1-\alpha) \rho_{o}$

The water content per kilogram in the tank is

$$
\beta=\frac{M_{w}}{M}=\frac{\rho_{w}}{\rho_{w}-\rho_{o}}\left(1-\frac{\rho_{o}}{\rho_{m}}\right)
$$

Where:

$\beta$ stands for T the water content per kilogram in crude oil storage tanks;

$M$ stands for the weight of oil-water mixture in crude oil storage tanks

In order to realize the measurement of crude oil in the tank, the moisture content in the crude oil storage tank must be calculated. That means the oil-water mixture density, pure oil density and water density in different temperatures must be calculated ${ }^{[3]}$.

Oil-water mixture density value can be obtained indirectly: by measuring the pressure difference from the liquid level to the bottom of the tank with a pressure difference transmitter, using ultrasonic level sensor to measure the tank liquid level, and then through the following formula

$$
\Delta P=\rho_{m} g H
$$

Where:

$\Delta P$ stands for the pressure difference from the liquid level to the bottom of

the tank

$\mathrm{H}$ stands for tank liquid level.

Then comes

$$
\rho_{m}=\frac{\Delta P}{g H}
$$

In different temperatures, there is a linear functional relation between the density and the temperature of the pure oil, the functional relationship between them is as follows

$$
\rho_{o}=\rho_{20}-\gamma(t-20)
$$

Where:

$\rho_{o}$ is the density of pure oil $\left(\mathrm{g} / \mathrm{cm}^{3}\right)$ when the temperature is $\mathrm{t}{ }^{\circ} \mathrm{C}$

$\rho_{20}$ is the density of pure oil at standard atmospheric pressure when it is $20{ }^{\circ} \mathrm{C}$, making this as the standard value of the density of pure oil $\left(\mathrm{g}^{\mathrm{cm}} \mathrm{cm}^{3}\right)$

$\gamma$ is the density temperature coefficient of pure oil.

Density values of water in different temperatures can be learned by querying Chemical Industry Handbook.

In summary, theory shows that, when the density of pure oil, pure water oil-water mixture in the tanks can be obtained, the moisture content of the the tank can be calculated through the formula, the volume of pure water of the tank can also be obtained, the volume of pure divides the cross sectional area equals the height of the water layer. After calculating the volume of pure oil of the 
tank, divide the cross sectional area of the tank, then the height of pure oil of the tank could be obtained, thus, the measurement of crude oil in the tanks is realized ${ }^{[4]}$.

\section{Design of Experimental Models}

Selection of the tank shape: actually, there are many shapes of the tank, mostly are cylindrical. After the analysis, the shape of oil tank does not have any impact on the experimental research. Therefore, from an economic and practical aspect, this paper uses a square tank as a model of crude oil storage tanks.

Selection of the material for the tank model: currently, the commonly used material for oil tanks is steel. For this experiment, material selection does not affect the experimental results. The heavy weight of steel cans makes it inconvenient to move and it is not easy to process mounting holes in the steel tank. From an economic and practical aspect, this experiment uses plexiglass plate as material of the tank model. Because plexiglass panels not only have good stiffness and strength, fully meet the conditions required for the experiment, it is light and inexpensive.

Selection of the size of the tank model: the actual height of the oil tank ranges from $7 / 8$ meters to tens of meters. The diameter of the tank is at least two to three meters. For such a tank size, laboratory condition is simply not available. Therefore, the only way is to make the size of the tank model as large as possible to close to the size of crude oil storage tank on the field.

In summary, the crude oil storage tank model is shown in Figure.1

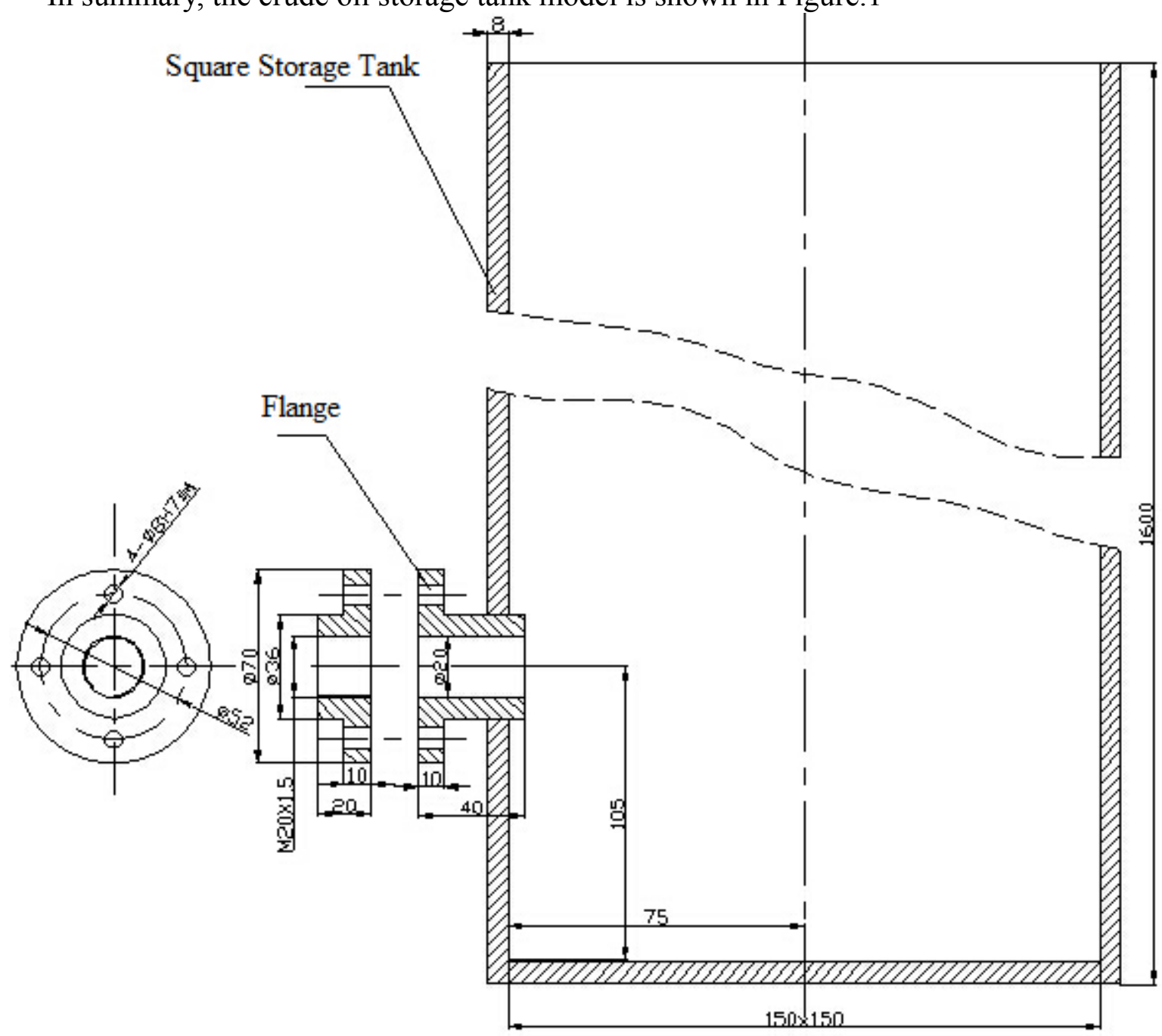

Figure. 1 The crude oil storage tank model 


\section{Selection of Pressure Difference Transmitter and Ultrasonic Sensors}

Pressure difference transmitter is a sensor making Industrial standard signal (e.g. standard voltage and current signal) as the output signal. It is mainly used to detect density, pressure and liquid level of liquids, gases and vapors. Through a detailed understanding of the performance, features and application fields of various types of pressure difference transmitter, as well as a comprehensive analysis of the measurement accuracy that should be met by it in the measurement system and other aspects including the range satisfy conducted this experiment selects the CYB13 pressure difference transmitter of Xi' an Xin Min Technology Limited ${ }^{[1]}$.

When the ultrasonic wave is propagating in a liquid, it has a good directivity and little energy loss since it can reflect when encountering interface. may be an ultrasonic reflection. Based on these characteristics of the ultrasonic wave, when the time difference measured from the moment ultrasonic wave was transmitted until it is received after the reflection is measured, the height of liquid can be calculated. In this experiment, RISEN-BS ultrasonic liquid level transmitter of Yi Zhao Technology Limited is used.

\section{Summary}

The method can accurately measure the liquid level of multiphase medium in the tank and crude oil production, it can be widely applied to various types of crude oil storage tanks, but influenced by temperature, it can not be used in low-temperature environment where is too cold that the liquid within the tank will be frozen.

This paper is subsided by Xianyang Normal University special project funding.

\section{References:}

[1] Zhang Hongmin. The Oil Market and Oil Finance [M]. Beijing: China Financial Publishing House, 2009: 16-20.

[2] Li Guozhen, Xiao Hua, Dong Shouping. oil-water separation technology and its progress [J] Oil and Gas Field Surface Engineering, 2011,20 (2): 7-9.

[3] Chen Xiangguang. Research on Applications Crude Oil Tank Level Detection Technology [J] Instrument Technique and Sensor, 2011 (05): 40-42.

[4] Ross Mistry, Chris Amaris, Alec Minty. sql server 2005 management and administration [M]. Imprint of Simon and Schuster 201 W.103 St.Indianapolis, IN USA, 2007: 64-70. 